\title{
New Insights on Output Capacitance Losses in Wide-Band-Gap Transistors
}

\author{
Mohammad Samizadeh Nikoo, Member, IEEE, Armin Jafari, Nirmana Perera, Student Member, IEEE, \\ and Elison Matioli, Member, IEEE
}

\begin{abstract}
The low ON-resistance of wide-band-gap (WBG) transistors is a key feature for efficient power converters, however, the anomalous loss in their output capacitance $\left(C_{0}\right.$ ss) severely limits their performance at high switching frequencies. Characterizing Coss-losses based on large-signal measurement methods requires an extensive effort, as separate measurements are needed at different operation points, including voltage-swing, frequency, and $\mathrm{d} v / \mathrm{d} t$. Furthermore, there is a practical trade-off in the maximum voltage and frequency applied to the device. Here we introduce a new circuit model, including an effective $C$ oss and a frequency-dependent series-resistance, along with a simple small-signal method to fully characterize Coss-losses in WBG transistors. The method accurately predicts $C$ oss-losses at any voltage-swing or frequency. Contrary to other methods, this technique directly leads to a general identification of $C$ oss-losses at different operation points, revealing new insights on $C_{\text {oss-losses in }}$ WBG transistors, especially the dependence of $E_{\text {DIss on voltage }}$ and frequency. Based on the proposed approach, the issue of $C_{\text {oss- }}$ losses in enhancement-mode $\mathrm{GaN}$ and $\mathrm{SiC}$ transistors was assigned to the limited quality-factor of $C$ oss. The precise

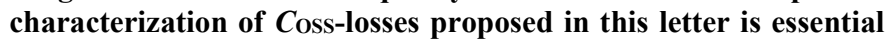
for designing efficient high-frequency power converters.
\end{abstract}

Index Terms-Output capacitance, Coss, Energy loss, EDIss, Nonlinear resonance, Superjunction, $\mathrm{SiC}, \mathrm{GaN}$, Cascode.

\section{INTRODUCTION}

$\mathrm{T}$ HE non-recoverable energy loss associated with resonantly charging and discharging the output capacitance ( $\left.C_{\mathrm{OSS}}\right)$ of some of the advanced transistors and diodes considerably limit their performance in power converters, especially those operating at high frequencies [1]-[11]. An unexpected power loss in soft-switched power converters based on $\mathrm{Si}$ superjunction (SJ) MOSFETs, especially those with low specific $R_{\mathrm{ON}}$, initiated studies on their large-signal $C_{\mathrm{OSs}}$, where a non-symmetric charging and discharging processes was observed. Measurements with Sawyer-Tower (ST) method showed a frequency-independent $C_{\text {Oss-loss due to the charge- }}$ trapping in SJ devices [8]-[11].

The lower-than-expected efficiencies in soft-switching power converters based on wide-band-gap (WBG) transistors, again initiated investigations on $C_{\text {oss- }}$ losses [12]. Zulauf et al. characterized $C_{\text {OSS }}$ charging/discharging energy dissipation ( $E_{\text {DISS }}$ ) in GaN transistors using Sawyer Tower (ST) method, where the results showed frequency-dependent losses [2].

The authors are with the Power and Wide-band-gap Electronics Research Laboratory (POWERlab), École polytechnique fédérale de Lausanne (EPFL), CH-1015 Lausanne, Switzerland (e-mail: elison.matioli@epfl.ch, mohammad.samizadeh@epfl.ch).
Guacci et al. used a thermal approach to study $C_{\text {oss-losses in }}$ $\mathrm{GaN}$ transistors and observed $\mathrm{d} v / \mathrm{d} t$-dependent energy dissipation [3]. The ST-based $E_{\text {DIss }}$ measurement of some of the commercial $\mathrm{SiC}$ transistors, however, presented a weak dependence on frequency and $\mathrm{d} v / \mathrm{d} t[1]$.

Although several measurements showed an effect from voltage-swing, frequency and $\mathrm{d} v / \mathrm{d} t$ on $C_{\text {Oss }}$-losses in $\mathrm{SiC}$ and enhancement-mode GaN transistors, the dependence of $E_{\text {DIss }}$ on these parameters is still not clear [3]. Zulauf et al. [1] used the empirical relation

$$
E_{\mathrm{DISS}}^{\mathrm{Ref}[1]}=k \cdot f^{\alpha} \cdot V^{\beta}
$$

to fit the experimental data, where $f$ is the frequency, $V$ is the charging voltage, and $k, \alpha$, and $\beta$ are constants. For some of the evaluated transistors, values of $\alpha<1$ and $\beta<2$ were obtained [13]. Although curve fitting from large-signal measurements

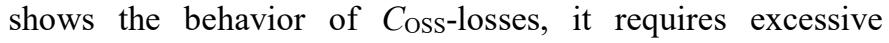
experiments with high-voltage and high-frequency power amplifiers at several operation points at different voltages and frequencies. Moreover, the need for a high-voltage RF power amplifier (PA) can severely limit the maximum voltage and frequency applied to the device under test (DUT).

In this work we propose a small-signal modeling approach to

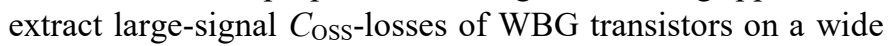
range of operation points. The device is simply modeled by a nonlinear capacitance $C_{\text {OSS }}$ in series with a frequencydependent resistance $R_{\mathrm{S}}$. This modeling decouples the frequency and voltage dependence of losses, enabling to solve a large-signal problem with a small-signal approach. Using an impedance measurement to measure values of $R_{\mathrm{S}}$ at different frequencies, together with the presented output capacitance versus voltage from datasheets, we present a general relationship for $C_{\text {oss-losses in different voltages and }}$ frequencies. As a result, just one simple small-signal measurement (instead of several different measurements in large-signal methods) leads to a general view of the $C_{\text {oss-losses. }}$ The method does not suffer from some of the shortcomings of large-signal measurements such as limitation in applied voltage/frequency (e.g. due to power amplifier), or signal distortion at high-frequencies. The proposed method gives insights on dependence of $E_{\mathrm{DISS}}$ on frequency, voltage, and $\mathrm{d} v / \mathrm{d} t$ value.

\section{MODEL}

Fig. 1(a) shows a model for output capacitance of transistors, including a nonlinear capacitance $C_{\mathrm{OSS}}$ in series with resistance 
$R_{\mathrm{S}}$ and in parallel with resistance $R_{\mathrm{P}}$, representative for losses at high and low frequencies, respectively. The quality-factor $(Q-$ factor) of output capacitance can be defined as [see Figs. 1(b) and (c)]

$$
Q=\frac{1}{R_{\mathrm{S}} C_{\mathrm{OSS}} \omega+\left(R_{\mathrm{P}} C_{\mathrm{OSS}} \omega\right)^{-1}}
$$

The effect of $R_{\mathrm{P}}$ (mainly corresponding to the leakage current) is dominant at $\mathrm{DC}$, while $R_{\mathrm{S}}$ significantly contributes to the

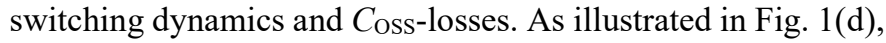
small-signal measurements show a considerable lossy behavior for $C_{\mathrm{Oss}}$ of WBG transistors. For instance, the levels of losses are considerably higher than a reference low-loss mica capacitor. The small-signal extracted values of losses for frequencies higher than $1 \mathrm{MHz}$, are in range of $1-10 \%$ which is in agreement with the previously measured large-signal losses [1], [2]. This indicates the possibility of evaluating large-signal losses with a proper small-signal modeling. Fig. 1(d) also shows that for frequencies higher than $1 \mathrm{MHz}$ (which covers the switching bandwidth of WBG transistors), the $Q$-factor of all the considered transistors is limited by $R_{\mathrm{S}}$.

For a linear capacitor with frequency-independent $R_{\mathrm{S}}$ and $R_{\mathrm{P}}$, (2) fully describes the charging/discharging energy dissipation as

$$
E_{\mathrm{DISS}}=\frac{\pi}{2 Q} E_{\mathrm{OSS}}
$$

where $E_{\text {OSs }}$ is the total energy stored in $C_{\text {OSs. }}$ In practice, however, $C_{\mathrm{OSs}}$ is nonlinear and $R_{\mathrm{S}}$ and $R_{\mathrm{P}}$ can change with
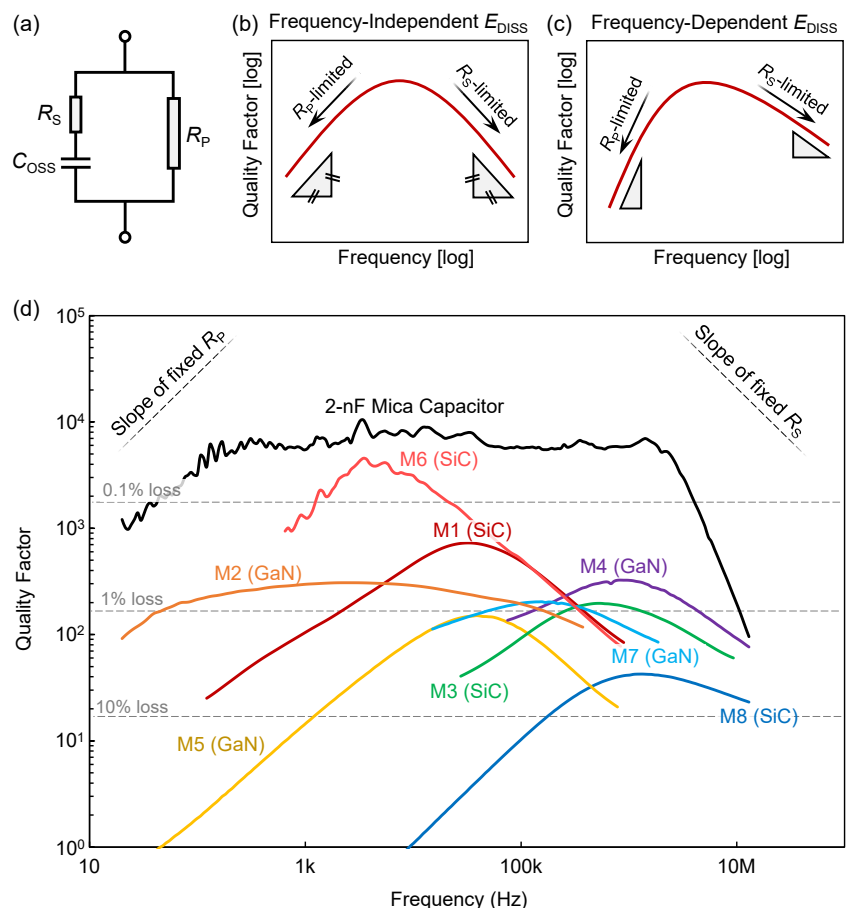

Fig. 1. (a) Circuit model for output capacitance of transistors. Quality-factor versus frequency for transistors with (b) fixed, and (c) frequency-dependent $R_{\mathrm{S}}$ and $R_{\mathrm{P}}$. (d) Measured quality-factor of $C_{\mathrm{OSs}}$ of different commercial WBG transistors versus frequency at $V_{\mathrm{DS}}=40 \mathrm{~V}$. The gate and source of transistors were shorted $\left(C_{\mathrm{OSS}}=C_{\mathrm{DS}}+C_{\mathrm{GD}}\right)$.
TABLE I

\begin{tabular}{|c|c|c|c|}
\hline \multirow{2}{*}{ No. } & \multicolumn{2}{|c|}{ Voltage and current rating } & \multirow{2}{*}{$\begin{array}{c}\mathrm{C}_{\mathrm{OSS}}^{* *} \\
(\mathrm{pF})\end{array}$} \\
\hline & Voltage (V) & Current $^{*}(\mathrm{~A})$ & \\
\hline M1 & 1200 & 36 & 80 \\
\hline M2 & 650 & 30 & 65 \\
\hline M3 & 650 & 93 & 118 \\
\hline M4 & 600 & 31 & 72 \\
\hline M5 & 100 & 90 & 840 \\
\hline M6 & 1700 & 4 & 16 \\
\hline M7 & 600 & 13 & 28 \\
\hline M8 & 1200 & 55 & 76 \\
\hline
\end{tabular}

SPECIFICATIONS OF EVALUATED WBG TRANSISTORS

* Continuous current at $25^{\circ} \mathrm{C}$.

** Reported capacitance at $2 / 3$ of voltage rating, measured at $1 \mathrm{MHz}$.

frequency [see Figs. 1(b) and 1(c)]. The non-unity slope of the $Q$-factor versus frequency for several different WBG transistors (Table I), shown in Fig. 1(d) (measured with Keysight E4990A impedance analyzer, with a very high accuracy), confirms the frequency-dependent nature of $R_{\mathrm{S}}$ and $R_{\mathrm{P}}$.

We use the model presented in Fig. 1(a) to extract $C_{\text {OSS }}$ charging /discharging energy dissipation. As mentioned, $R_{\mathrm{S}}$ is the origin of $C_{\mathrm{OSs}}$-losses in switching dynamics, as $R_{\mathrm{P}}$ just limits the $Q$-factor at low frequencies. By applying voltage $v(t)$ to the output capacitance, and considering $R_{\mathrm{S}}$ as a perturbation element, the power loss in $R_{\mathrm{S}}$ can be written as

$$
P_{\text {loss }}=R_{\mathrm{S}}\left(C_{\mathrm{OSs}} \frac{\mathrm{d} v}{\mathrm{~d} t}\right)^{2}
$$

Assuming $v(t)$ represents a switching transient from 0 to $V$, the total energy loss during a single switching transient time $t_{\mathrm{SW}}$ is

$$
E_{\text {loss }}=\int_{0}^{t_{\mathrm{Sw}}} R_{\mathrm{S}}\left(C_{\text {Oss }} \frac{\mathrm{d} v}{\mathrm{~d} t}\right)^{2} \mathrm{~d} t
$$

In a charging and discharging process, however, $E_{\text {loss }}$ is dissipated two times ( $\left.E_{\text {DISS }}=2 E_{\text {loss }}\right)$. Considering a constant switching-speed $\mathrm{d} v / \mathrm{d} t \cong V / t_{\mathrm{SW}}$, which is very accurate for trapezoidal waveforms and also can be used for sinusoidal waveforms, we write

$$
E_{\text {DISS }}=2 R_{\mathrm{S}}\left(\frac{\mathrm{d} v}{\mathrm{~d} t}\right) \int_{0}^{V} C_{\mathrm{OSS}}^{2} \mathrm{~d} v
$$

which clearly shows $\mathrm{d} v / \mathrm{d} t$-dependence of $C_{\text {oss-losses [2]-[4]. }}$ Equation (6) can be rewritten as

$$
E_{\text {DISS }}=2 R_{\mathrm{S}}\left(\frac{\mathrm{d} v}{\mathrm{~d} t}\right) V C_{\mathrm{OSS}}^{\text {eff } 2}
$$

in which we introduced the new term $C_{\mathrm{OSS}}^{\mathrm{eff}}$ which is the root mean square (rms) of $C_{\mathrm{Oss}}$ from 0 to $V$, representing the average $C_{\mathrm{OSs}}$ value that contributes to power dissipation in the device output capacitance

$$
C_{\mathrm{OSS}}^{\mathrm{eff}}=\sqrt{\frac{1}{V} \int_{0}^{V} C_{\mathrm{OSS}}^{2} \mathrm{~d} v}
$$

One can use $f=1 /\left(2 t_{\mathrm{sW}}\right)$ to rewrite (6) as

$$
E_{\text {DISS }}=4 R_{\mathrm{S}} f V^{2} C_{\text {OSS }}^{\text {eff } 2}
$$

Comparing (9) with the experimental model (1) reveals two main points:

(C) 2019 IEEE. Personal use of this material is permitted. Permission from IEEE must be obtained for all other uses, in any current or future media, including reprinting/republishing this material for advertising or promotional purposes, creating new collective works, for resale or redistribution to servers or lists, or reuse of any copyrighted component of this work in other works. 
1) $\alpha=1$ for a fixed and frequency-independent $R_{\mathrm{S}}$, however, non-unity values of $\alpha$ have been reported in [2] and [13]. This agrees with the measurement results presented in Fig. 1(d), showing frequency-dependent $R_{\mathrm{S}}$.

2) The obtained values of $\beta$, extracted by curve fitting in [13], were always less than 2 . This is in agreement with (9) since when rising the voltage $V$, the rms value of $C_{\mathrm{OSS}}$ decreases. As a result, although square of $V$ is seen in (9),

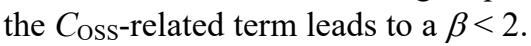

\section{Coss-Loss Evaluation}

Here we show how large-signal $C_{\text {Oss-losses can be extracted }}$ from the small-signal model. This section also validates the applicability of the proposed method to evaluate $C_{\text {OSs-losses, }}$ by comparing the extracted $E_{\text {DISS }}$ values with the ST method [1], [2]. The first step to extract the general relation of (9) is to obtain the effective $C_{\text {oss. }}$. This can be done by using data reported in datasheets. Figs. 2(a) and (b) show the reported $C_{\text {oss }}$ in datasheet of transistors M1 (36-A-rated SiC FET with $R_{\mathrm{ON}}=$ $80 \mathrm{~m} \Omega$ ) and M2 (30-A-rated e-mode GaN HEMT with $R_{\mathrm{ON}}=$ $50 \mathrm{~m} \Omega$ ), respectively. the effective $C_{\mathrm{OSs}}$ values were obtained using (8) [see Figs. 2(a) and (b)]. One can directly use these values, however, here we applied a curve fitting to obtain closed-form relations [dashed lines in Figs. 2(a) and (b)]:

$$
C_{\mathrm{OSS}}^{\mathrm{eff}}=(2850 \mathrm{pF}) \times(V[\mathrm{~V}])^{-0.38}
$$

and

$$
C_{\mathrm{OSS}}^{\mathrm{eff}}=(2350 \mathrm{nF}) \times(V[\mathrm{~V}])^{-0.42}
$$

for M1 and M2, respectively.

After extraction of the effective $C_{\mathrm{OSS}}$, the series resistance $R_{\mathrm{S}}$ was measured using a Keysight E4990A impedance analyzer [see Fig. 2(c)]. The $R_{\mathrm{S}}$, as a key element in $C_{\text {oss-losses, as a }}$ function of frequency is not typically reported by manufacturers
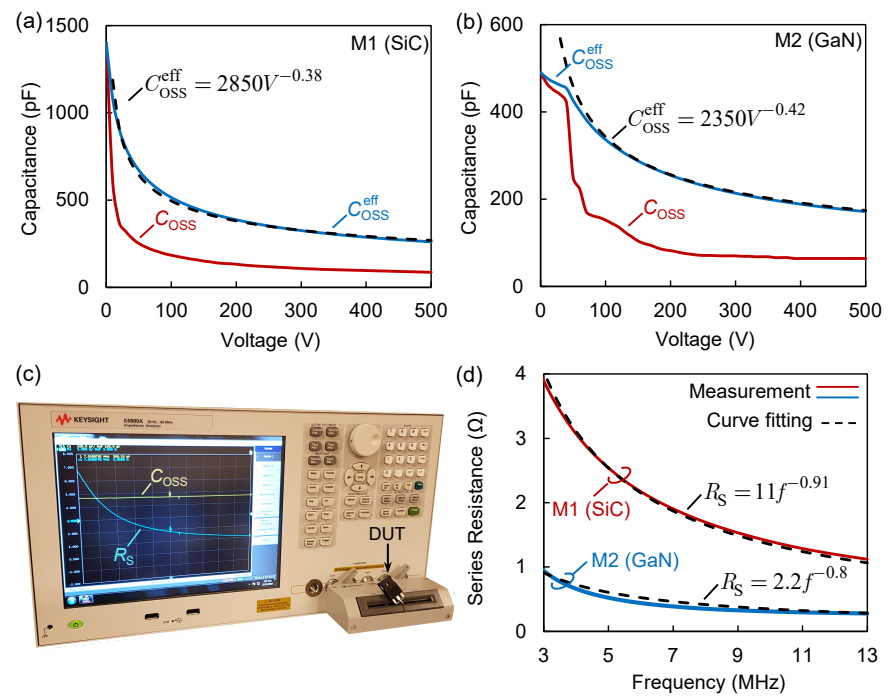

Fig. 2. $\quad C_{\text {Oss }}$ (at $1 \mathrm{MHz}$, reported in datasheet) and extracted effective $C_{\text {OSS }}$ for (a) M1 (SiC), and (b) M2 (GaN). (c) Photograph of the experimental set-up for measuring $R_{\mathrm{S}}$ (the gate and drain of the transistor are shorted) using Keysight E4990A impedance analyzer. (d) $R_{\mathrm{S}}$ as a function of frequency for M1 and M2 $\left(V_{\mathrm{DS}}=40 \mathrm{~V}\right)$. Measurement (solid line) and curve fitting (dashed line).
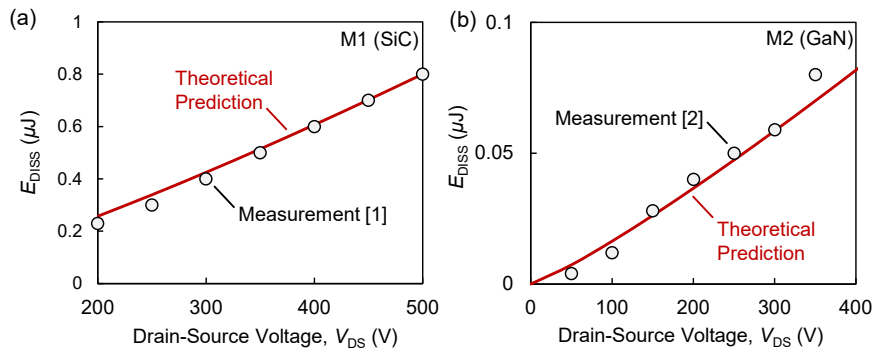

Fig. 3. Theoretical prediction of $C_{\text {oss }}$ energy dissipation (solid lines) compared with measurement results with ST method [1], [2] for (a) M1 (at 1 MHz) (b) M2 (at $10 \mathrm{MHz}$ ).

in datasheets. Fig. 2(d) illustrates the $R_{\mathrm{S}}$ for transistors M1 and M2 (solid lines). Unlike $C_{\text {oss, }}$ which is strong function of voltage, $R_{\mathrm{S}}$ is almost constant with voltage; and therefore it can be assumed as linear parameter. On the other hand, $C_{\text {oss }}$ is not a function of frequency, while $R_{\mathrm{S}}$ is highly frequencydependent [see Fig. 2(d)]. For M1 and M2 we have

$$
R_{\mathrm{S}}=(11 \Omega) \times(f[\mathrm{MHz}])^{-0.91}
$$

and

$$
R_{\mathrm{S}}=(2.2 \Omega) \times(f[\mathrm{MHz}])^{-0.8}
$$

respectively. It should be noted that the level of $R_{\mathrm{S}}$, as the effective series resistance of $C_{\text {OSs }}$, is significantly higher than the device on-resistance (e.g. in GaN devices it includes the buffer and Si substrate instead of the two-dimensional electron gas [14]). Replacing (10)-(13) into (9) results in

$$
E_{\text {DISS }}=0.357 \times f^{0.09} \times V^{1.24}[\mathrm{~nJ}], \text { for M1 (SiC) }
$$

and

$$
E_{\text {DISS }}=0.049 \times f^{0.2} \times V^{1.16}[\mathrm{~nJ}], \text { for M2 }(\mathrm{GaN})
$$

where $f$ and $V$ are in $\mathrm{MHz}$ and Volts, respectively, showing losses at different operation points just by performing two measurements. The obtained relations were verified with measurement results using ST method with sinusoidal waveforms performed at $1 \mathrm{MHz}$ for $\mathrm{M} 1$ [1] and at $10 \mathrm{MHz}$ for M2 [2]. As shown in Fig. 3, good agreements were obtained for both transistors. It should be noted that the level of losses in these transistors are considerably different, showing the applicability of the proposed approach to extract $C_{\text {Oss-losses for }}$ a wide range of $E_{\mathrm{DISS}}$ values.

\section{DISCUSSION}

Despite several experimental works on $C_{\text {Oss-losses in WBG }}$ transistors, the dependence of $E_{\mathrm{DISS}}$ on frequency is not completely clear. Some transistors showed strong dependence of $E_{\mathrm{DISS}}$ on frequency, while for some other transistors it was very weak. The same observations can be seen for $\mathrm{d} v / \mathrm{d} t$ dependence. For some transistors, even lower $E_{\text {DISS values were }}$ obtained for higher $\mathrm{d} v / \mathrm{d} t$ values [1].The proposed approach, however, clears the dependence of $E_{\text {DISs }}$ on the frequency and $\mathrm{d} v / \mathrm{d} t$.

Based on (9) $E_{\text {DISS }} \sim R_{\mathrm{S}}(f) \times f$, which shows the frequency-

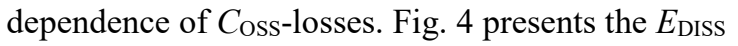

(C) 2019 IEEE. Personal use of this material is permitted. Permission from IEEE must be obtained for all other uses, in any current or future media, including reprinting/republishing this material for advertising or promotional purposes, creating new collective works, for resale or redistribution to servers or lists, or reuse of any copyrighted component of this work in other works. 


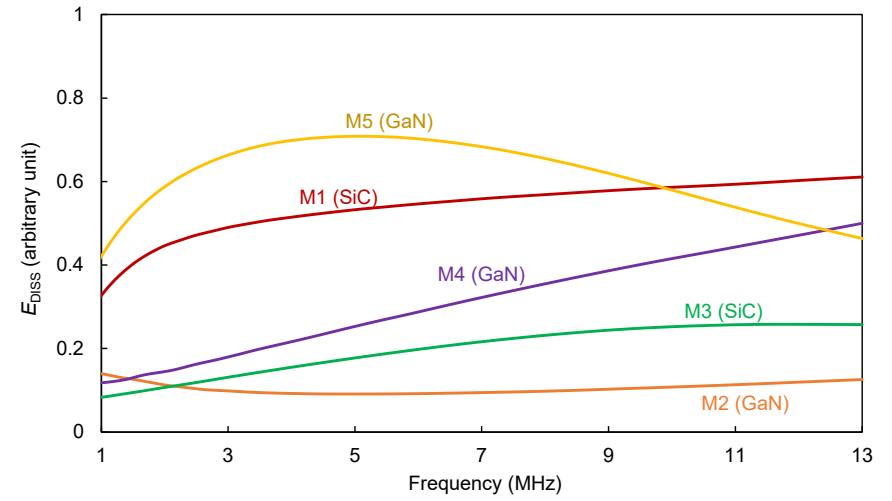

Fig. 4. Frequency-dependence of $E_{\text {DIss }}$ obtained from the proposed model versus frequency for five $\mathrm{SiC}$ and $\mathrm{GaN}$ transistors.

dependence on frequency for several different transistors extracted using this model. $R_{\mathrm{S}}$ values were measured at $40 \mathrm{~V}$. Since $R_{\mathrm{S}}$ shows negligible variation with voltage, the obtained results can be generalized to device performance at different voltages. Transistor M1 $(\mathrm{SiC})$ shows a saturation in $E_{\mathrm{DISs}}$ versus frequency, in agreement with measurement results in [1], which showed an almost frequency-independent $E_{\mathrm{DISS}}$. Transistors M2 $(\mathrm{GaN})$ and $\mathrm{M} 3(\mathrm{SiC})$ show almost constant $E_{\mathrm{DISS}}$ for frequencies higher than $5 \mathrm{MHz}$. The experimental results in [2] also present almost equal losses at $5 \mathrm{MHz}$ and $10 \mathrm{MHz}$, for M2. Transistor M4, however, shows a significant increase in $E_{\mathrm{DISS}}$, at higher frequencies $(>1 \mathrm{MHz})$. This is similar to most typical cases in the reported experimental results [2], [13]. Interestingly, for transistor M5, there is an initial increase in $E_{\text {DISS, however, after }}$ $f=5 \mathrm{MHz}$, the losses even decrease with increasing the frequency. The similar behavior has been reported for some of $\mathrm{SiC}$ transistors, showing lower $E_{\mathrm{DISS}}$ at higher frequencies or higher $\mathrm{d} v / \mathrm{d} t$ values. As a conclusion, different frequencydependence types can be observed in $E_{\text {DISS }}$ in WBG transistors, and the present method allows to investigate such effects using a small-signal modeling.

The proposed method gives a complete view of $C_{\text {oss-losses }}$ due to limited $Q$-factor in $\mathrm{SiC}$ and enhancement-mode $\mathrm{GaN}$ transistors. Here we separately discuss about applicability of the method for two other types of devices:

1) SJ devices: The dominant part of Coss-loss in SJ transistors is due to the charge-trapping which causes frequency-independent energy dissipation. SJ devices, however, can potentially have a limited $Q$-factor. In this case, a frequency-dependent loss is added to the total energy dissipation. This might be the reason of previously observed frequency-dependent losses in some of SJ transistors [16]. In this case, a low frequency ST measurement (frequencyindependent losses) together with the proposed method (frequency-dependent losses) give a complete view of $C_{\text {oss }}$ energy dissipation.

2) Cascode devices: A type of frequency-independent losses, different from that is happening for SJ devices, was observed in cascode transistors [4], [15]. Some of the commercial cascode
GaN (integrated with a low-voltage Si device) devices showed considerably higher loss for voltages larger than $\sim 200 \mathrm{~V}$ [2], [4]. As a result, one can separately characterize frequencyindependent (using ST method at the frequency corresponding to peak of $Q$-factor) and frequency-dependent (using the proposed method) Coss-losses. Adding these different component gives the general behavior of energy dissipation in the output capacitance.

Based on the proposed model, it is suggested to manufacturers to present $R_{\mathrm{S}}$-versus-frequency (at least for frequencies above $1 \mathrm{MHz}$ ) for WBG transistors. This curve, together with $C_{\mathrm{OSS}}^{\mathrm{eff}}$ gives a general view on $C_{\mathrm{Oss}}$-losses.

\section{CONCLUSION}

We proposed a new method to extract $C_{\text {Oss-losses for WBG }}$ transistors at different voltages and time/frequency frames, just

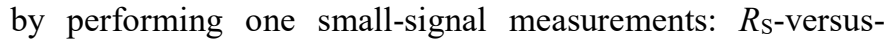
frequency. This measurement together with the reported $C_{\text {oss- }}$

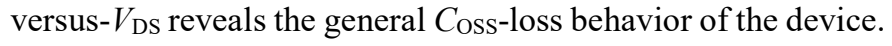
The method helps to clear the voltage and frequency dependence of $E_{\mathrm{DISS}}$, and can be used to compare and benchmark different semiconductor devices. The results also led to a categorization of $C_{\text {oss-loss }}$ in different types of transistors. The $E_{\mathrm{DISS}}$ in e-mode $\mathrm{GaN}$ and $\mathrm{SiC}$ transistors is mainly caused by the limited $Q$-factor of $C_{\text {oss, }}$, which was not observed in SJ and cascode devices. The generality and robustness of this method make it possible to quantify $C_{\text {oss- }}$ losses of WBG transistors as a crucial source of losses in soft switch power converters, especially those operating at high frequencies.

\section{REFERENCES}

[1] G. Zulauf, Z. Tong, J. D. Plummer, and J. M. Rivas-Davila, "Active power device selection in high- and very-high-frequency power converters," IEEE Trans. Power Electron., vol. 34, no. 7, pp. 6818-6833, Jul. 2019.

[2] G. Zulauf, S. Park, W. Liang, K. Surakitbovorn, and J. Rivas-Davila, " $C_{\text {oss }}$ losses in $600 \mathrm{~V} \mathrm{GaN}$ power semiconductors in soft-switched, highand very-high-frequency power converters," IEEE Trans. Power Electron., vol. 33, no. 12, pp. 10748-10763, Dec. 2018.

[3] M. Guacci, et al. "On the origin of the $C_{\mathrm{Oss}}$-losses in soft switching GaNon-Si power HEMTs," IEEE J. Emerg. Sel. Topics Power Electron., vol. 7, no. 2, pp. 679-694, Jun. 2019.

[4] M. S. Nikoo, A. Jafari, N. Perera, E. Matioli, "Measurement of largesignal $C_{\text {oss }}$ and $C_{\text {oss }}$ losses of transistors based on nonlinear resonance," IEEE Trans. Power Electron., early access, 2019.

[5] D. Bura, T. Plum, J. Baringhaus, and R. W. De Doncker, "Hysteresis Losses in the Output Capacitance of Wide Bandgap and Superjunction Transistors," in 2018 20th European Conference on Power Electronics and Applications (EPE'18 ECCE Europe), Sep. 2018, pp. P.1-P.9.

[6] D. Rothmund, D. Bortis, and J. W. Kolar, "Accurate transient calorimetric measurement of soft-switching losses of $10-\mathrm{kV}$ SiC MOSFETS and diodes," IEEE Trans. Power Electron., vol. 33, no. 6, pp. 5240-5250, Jun. 2018.

[7] Z. Tong, G. Zulauf, J. Xu, J. D. Plummer, and J. Rivas-Davila, "Output capacitance loss characterization of silicon carbide schottky diodes," IEEE Trans. Power Electron., IEEE J. Emerg. Sel. Topics Power Electron., vol. 7, no. 2, pp. 856-878, Jun. 2019. 
[8] G. D. Zulauf, J. Roig-Guitart, J. D. Plummer, and J. M. Rivas-Davila, " $C_{\mathrm{OSS}}$ measurements for superjunction MOSFETs: Limitations and opportunities," IEEE Trans. Electron Devices, vol. 66, no. 1, pp. 578584, Jan. 2019.

[9] J. Fedison and M. Harrison, " $C_{\mathrm{Oss}}$ hysteresis in advanced superjunction MOSFETs," Appl. Power Electron. Conf. Expo., 2016, pp. 247-252.

[10] J. Fedison, M. Fornage, M. Harrison, and D. Zimmanck, " $\mathrm{C}_{\text {oss }}$ related energy loss in power MOSFETs used in zero-voltage-switched applications," in Proc. 29th Annu. IEEE Appl. Power Electron. Conf. Expo., 2014, pp. 150-156.

[11] J. Roig and F. Bauwens, "Origin of anomalous $C_{\text {oss }}$ hysteresis in resonant converters with superjunction FETs," IEEE Trans. Electron Devices, vol. 62, no. 9, pp. 3092-3094, Sep. 2015.

[12] K. Surakitbovorn and J. R. Davila, "Evaluation of GaN transistor losses at $\mathrm{MHz}$ frequencies in soft switching converters," in Proc. IEEE Control Model. Power Electron., 2017, pp. 1-6.

[13] K. Surakitbovorn and J. R. Davila, "On the Optimization of a Class-E Power Amplifier with GaN HEMTs at MHz Operation," IEEE Trans. Power Electron., early access, 2019.

[14] J. Zhuang, G. Zulauf, J. Roig, J. D. Plummer, J. Rivas-Davila, “An Investigation into the Causes of Coss Losses in GaN-on-Si HEMTs," in Proc. IEEE 20th Workshop Control Modelling Power Electron. (COMPEL), Jun. 2019.

[15] J. Xu, L. Gu, S. Kargarrazi, D. Senesky, and J. Rivas-Davila, "Cascode $\mathrm{GaN} / \mathrm{SiC}$ power device for $\mathrm{MHz}$ switching," in Proc. 24th Annu. IEEE Appl. Power Electron. Conf. Expo., 2019.

[16] G. Zulauf and J. M. Rivas-Davila, "Coss losses in silicon superjunction MOSFETs across constructions and generations," in Proc. IEEE 30th Int. Symp. Power Semiconductor Devices ICs (ISPSD), May 2018, pp. 136139. 